\title{
Algebraic Cryptanalysis of McEliece Variants with Compact Keys
}

\author{
Jean-Charles Faugère ${ }^{1}$, Ayoub Otmani ${ }^{2,3}$, \\ Ludovic Perret ${ }^{1}$, and Jean-Pierre Tillich ${ }^{2}$ \\ 1 SALSA Project - INRIA (Centre Paris-Rocquencourt) \\ UPMC, Univ Paris 06 - CNRS, UMR 7606, LIP6 \\ 104, avenue du Président Kennedy 75016 Paris, France \\ jean-charles.faugere@inria.fr, ludovic.perret@lip6.fr \\ 2 SECRET Project - INRIA Rocquencourt \\ Domaine de Voluceau, B.P. 10578153 Le Chesnay Cedex, France \\ ayoub.otmani@inria.fr, jean-pierre.tillich@inria.fr \\ 3 GREYC - Université de Caen - Ensicaen \\ Boulevard Maréchal Juin, 14050 Caen Cedex, France
}

\begin{abstract}
In this paper we propose a new approach to investigate the security of the McEliece cryptosystem. We recall that this cryptosystem relies on the use of error-correcting codes. Since its invention thirty years ago, no efficient attack had been devised that managed to recover the private key. We prove that the private key of the cryptosystem satisfies a system of bi-homogeneous polynomial equations. This property is due to the particular class of codes considered which are alternant codes. We have used these highly structured algebraic equations to mount an efficient key-recovery attack against two recent variants of the McEliece cryptosystems that aim at reducing public key sizes. These two compact variants of McEliece managed to propose keys with less than 20,000 bits. To do so, they proposed to use quasi-cyclic or dyadic structures. An implementation of our algebraic attack in the computer algebra system MAGMA allows to find the secret-key in a negligible time (less than one second) for almost all the proposed challenges. For instance, a private key designed for a 256-bit security has been found in 0.06 seconds with about $2^{17.8}$ operations.
\end{abstract}

\section{Introduction}

Alternative cryptography. Despite the fact that several hard problems have been proposed as a foundation for public-key primitives, those effectively used are essentially classical problems coming from number theory: integer factorization (e.g. in RSA) and discrete logarithm (e.g. in Diffie-Hellman key-exchange). It is well-known that, although polynomial-time algorithms for those problems have not yet been found, they are not safe from a theoretic breakthrough that would endanger the security of the corresponding schemes. For instance, the emergence 
of a new computer model such as quantum computers would make schemes based on these classical number theory problems totally insecure.

The lack of diversity in public key cryptography has been identified as a major concern in the field of information security. A good illustration of the potential damage of such lack of diversity is hash zoo. The portfolio of hash functions used so far in practice was mainly restricted to the same type of functions which are now almost all broken. Although the American National Institute of Standards and Technology (NIST) issued an international call 11 to design a new standard hash function, the cryptography community will remain in a fuzzy situation until 2012 (date of final decision).

One of the main issues in public key cryptography is to identify hard problems that are not based on the classical ones coming from number theory. However, few emerged until now as a viable alternative. As pointed in [2, promising candidates include: the problem of solving multivariate equations over a finite field, the problem of finding a short vector in a lattice and the problem of decoding a linear code. Those problems known for being NP-hard are not concerned with the quantum computer threat.

McEliece cryptosystem. Among those problems, code-based cryptosystems seem to offer the most promising alternative. McEliece public key cryptosystem 25] is one of the oldest public-key cryptosystems. Its security relies on the difficulty of decoding a linear code. The main advantage of this system is to have very fast encryption and decryption functions. Depending on how the parameters are chosen for a fixed security level, this cryptosystem is about five times faster for encryption and about 10 to 100 times faster for decryption than RSA [10. Furthermore, it has withstood many attacking attempts. After more than thirty years now, it still belongs to the very few public key cryptosystems which remain unbroken.

Following McEliece's pioneering work, several different public key cryptosystems based on the intractability of decoding a linear code have been proposed 282031237643527. The original McEliece cryptosystem relies on Goppa codes whereas its variants suggested to use different codes. The Sidelnikov system 31] used Reed-Muller codes, the Janwa-Moreno system proposed to take algebraic geometric codes 23. and the Gabidulin-Paramonov-Tretjakov (GPT) cryptosystem considered Gabidulin codes devised for the rank-metric. LDPC codes have also been repeatedly suggested for this use. Niederreiter is the first in 28] to bring in a significant modification of the McEliece system. However his suggestion to use Generalized Reed-Solomon codes turned out to be an insecure solution [32]. Many of these schemes were broken [32 22 26 29 18 30 35]. All these attacks result in a total break of the system (the secret key, or an equivalent secret key is recovered from the knowledge of the public key). However, the original McEliece remains unbroken. The fact that the best known attacks are still exponential speaks for itself [338919].

${ }^{1}$ http://csrc.nist.gov/groups/ST/hash/sha-3/index.html 
Despite its impressive resistance against a variety of attacks and its fast encryption and decryption, McEliece cryptosystem has not stood up to RSA for practical applications. This is most likely due to the large size of the public key which is between several hundred thousand and several million bits. To overcome this limitation, a new trend initiated in 21] manages to decrease the key size by choosing the public matrix defining the code in a particular form; for instance with a quasi-cyclic structure [21]. This enables to decrease significantly the key size. The same idea was used in [4] with LDPC codes. Both schemes were broken in 29. It should be noted that both proposals did not use the Goppa codes of the McEliece cryptosystem, and the attacks have no impact on its security.

This work was then followed by two independent proposals [5 27] that are based on the same kind of idea of using quasi-cyclic [5] or dyadic structure [27. Both use the same type of codes called the alternant code family which contains Goppa codes. Actually the codes used in [27] are Goppa codes. This approach is quite attractive because it results in a drastic improvement of the public key size. In [5], the size ranges between 8,000 and 20,000 bits, whereas it lies between 4, 000 and 20,000 bits in [27. Until now, these new proposals seem to be immune against the attack suggested in [29].

Our contribution. In this paper we show that both schemes have a serious flaw that can be exploited to recover the private keys. We present an algebraic cryptanalysis 2 of the quasi-cyclic and dyadic schemes [5 27]. Algebraic cryptanalysis is a general framework that permits to assess the security of theoretically all cryptographic schemes. So far, such type of attacks has been applied successfully against several multivariate schemes and stream ciphers. To our knowledge, it is the first time that such an approach is used against code-based cryptosystems. The basic principle of this cryptanalysis is to associate to a cryptographic primitive a set of algebraic equations. The system of equations is constructed in such a way to have a correspondence between the solutions of this system, and a secret information of the cryptographic primitive (for instance the secret key of an encryption scheme). In McEliece, the algebraic system that we have to solve for recovering the secret-key has the following very specific structure:

$$
\left\{g_{i, 0} Y_{0} X_{0}^{j}+\cdots+g_{i, n-1} Y_{n-1} X_{n-1}^{j}=0 \mid i \in\{0, \ldots, k-1\}, j \in\{0, \ldots, r-1\}\right\}
$$

where the unknowns are the $X_{i}$ 's and the $Y_{i}$ 's and the $g_{i, j}$ 's are known coefficients with $0 \leq i \leq k-1,0 \leq j \leq n-1$ that belong to a certain field $\mathbb{F}_{q}$ with $q=2^{s}$. We look for solutions of this system in a certain extension field $\mathbb{F}_{q^{m}}$. Here $k$ is an integer which is at least equal to $n-r m$. By denoting $\mathbf{X} \stackrel{\text { def }}{=}\left(X_{0}, \ldots, X_{n-1}\right)$ and $\mathbf{Y} \stackrel{\text { def }}{=}\left(Y_{0}, \ldots, Y_{n-1}\right)$ we will refer to such an algebraic system by $\operatorname{McE}_{k, n, r}(\mathbf{X}, \mathbf{Y})$. The total number of equations is $r k$. The number of unknowns $2 n$ and the maximum degree $r-1$ of the equations can be extremely high when cryptographic

${ }^{2}$ An independent and parallel work 34 took place that also proposed a cryptanalysis of these two schemes. 
parameters are considered (e.g. $n=1024$ and $r-1=49$ ). Thus it is not clear whether an algebraic attack can be mounted efficiently in general.

However, in the case of the tweaked McEliece schemes we have either quasicyclic or dyadic [527]. It turns out that is possible to make use of this structure in order to reduce considerably the number of unknowns in the algebraic system (11). Moreover, it also appears that by using only the linear equations involving the $Y_{i}$ 's gives a linear space of solutions which is of small dimension. We will explain in Section 4 and Section 5 respectively how to solve the underlying algebraic systems. We will see how the public-key structure (quasi-cyclic or dyadic) impacts on the difficulty of solving the algebraic system (1). In particular, the structure induces an imbalance between the $X$ and $Y$ variables. From a practical point of view, we have been able to recover the secret-key via Gröbner bases computations in a negligible time (less than one second) for most of the parameters proposed in [5]27]. Before that, we briefly recall in the next section the McEliece scheme and we explain in Section 3 how we derive the algebraic system (11).

\section{McEliece Public-Key Cryptosystem}

We recall here how the McEliece public-key cryptosystem is defined.

Secret key: The triplet $\left(\boldsymbol{S}, \boldsymbol{G}_{\boldsymbol{s}}, \boldsymbol{P}\right)$ of matrices defined over a finite field $\mathbb{F}_{q}$ over $q$ elements, with $q$ being a power of two, that is $q=2^{s} . \boldsymbol{G}_{\boldsymbol{s}}$ is a full rank matrix of size $k \times n$, with $k<n, \boldsymbol{S}$ is of size $k \times k$ and is invertible, and $\boldsymbol{P}$ is permutation matrix of size $n \times n$. Moreover $\boldsymbol{G}_{\boldsymbol{s}}$ defines a code (which is the set of all possible $\boldsymbol{u} \boldsymbol{G}_{\boldsymbol{s}}$ with $\boldsymbol{u}$ ranging over $\mathbb{F}_{q}^{k}$ ) which has a decoding algorithm which can correct in polynomial time a set of errors of weight at most $t$. This means that it can recover in polynomial time $\boldsymbol{u}$ from the knowledge of $\boldsymbol{u} \boldsymbol{G}_{\boldsymbol{s}}+\boldsymbol{e}$ for all possible $e \in \mathbb{F}_{q}^{n}$ of Hamming weight at most $t$.

Public key: The matrix product $\boldsymbol{G}=\boldsymbol{S} \boldsymbol{G}_{\boldsymbol{s}} \boldsymbol{P}$.

Encryption: A plaintext $\boldsymbol{u} \in \mathbb{F}_{q}^{k}$ is encrypted by choosing a random vector $\boldsymbol{e}$ in $\mathbb{F}_{q}^{n}$ of weight at most $t$. The corresponding ciphertext is $\boldsymbol{c}=\boldsymbol{u} \boldsymbol{G}+\boldsymbol{e}$.

Decryption: $\boldsymbol{c}^{\prime}=\boldsymbol{c} \boldsymbol{P}^{-1}$ is computed from the ciphertext $\boldsymbol{c}$. Notice that $\boldsymbol{c}^{\prime}=$ $\left(\boldsymbol{u} \boldsymbol{S} \boldsymbol{G}_{\boldsymbol{s}} \boldsymbol{P}+\boldsymbol{e}\right) \boldsymbol{P}^{-1}=\boldsymbol{u} \boldsymbol{S} \boldsymbol{G}_{\boldsymbol{s}}+\boldsymbol{e} \boldsymbol{P}^{-1}$ and that $\boldsymbol{e} \boldsymbol{P}^{-1}$ is of Hamming weight at most $t$. Therefore the aforementioned decoding algorithm can recover in polynomial time $\boldsymbol{u} \boldsymbol{S}$. This vector is multiplied by $\boldsymbol{S}^{-1}$ to obtain the plaintext $\boldsymbol{u}$.

This describes the general scheme suggested by McEliece. From now on, we say that $\boldsymbol{G}$ is the public generator matrix and the vector space $\mathscr{C}$ spanned by its rows is the public code i.e. $\mathscr{C} \stackrel{\text { def }}{=}\left\{\boldsymbol{u} \boldsymbol{G} \mid \boldsymbol{u} \in \mathbb{F}_{q}^{k}\right\}$. What is generally referred to as the McEliece cryptosystem is this scheme together with a particular choice of the code, which consists in taking a binary Goppa code. This class of codes belongs to a more general class of codes, namely the alternant code family ([24, Chap. 12, p. 365]). The main feature of this last class of codes is the fact that they can be decoded in polynomial time. 


\section{Algebraic Approach}

Setting up the algebraic system. We explain more precisely how we construct the algebraic system described in (1). As explained in the previous section, the McEliece cryptosystem relies on Goppa codes which belong to the class of alternant codes and inherit from this an efficient decoding algorithm. We will describe this class of codes in more details since both cryptosystems that we cryptanalyze use such codes. It is convenient to describe them through a paritycheck matrix. This is an $r \times n$ matrix $\boldsymbol{H}$ defined over an extension $\mathbb{F}_{q^{m}}$ of the field over which the code is defined, which is such that

$$
\left\{\boldsymbol{u} \boldsymbol{G}_{\boldsymbol{s}} \mid \boldsymbol{u} \in \mathbb{F}_{q}^{k}\right\}=\left\{\boldsymbol{c} \in \mathbb{F}_{q}^{n} \mid \boldsymbol{H} \boldsymbol{c}^{T}=0\right\} .
$$

$r$ satisfies in this case the condition $r \geq \frac{n-k}{m}$. In the case of alternant codes, there exists a parity-check matrix with a very special form related to Vandermonde matrices. More precisely there exist two vectors $\boldsymbol{x}=\left(x_{0}, \ldots, x_{n-1}\right)$ and $\boldsymbol{y}=$ $\left(y_{0}, \ldots, y_{n-1}\right)$ in $\mathbb{F}_{q^{m}}^{n}$ such that $\boldsymbol{V}_{r}(\boldsymbol{x}, \boldsymbol{y})$ is a parity-check matrix, with

$$
\boldsymbol{V}_{r}(\boldsymbol{x}, \boldsymbol{y}) \stackrel{\text { def }}{=}\left(\begin{array}{lll}
y_{0} & \cdots & y_{n-1} \\
y_{0} x_{0} & \cdots & y_{n-1} x_{n-1} \\
\vdots & & \vdots \\
y_{0} x_{0}^{r-1} & \cdots & y_{n-1} x_{n-1}^{r-1}
\end{array}\right)
$$

We use the following notation in what follows

Definition 1. The alternant code $\mathscr{A}_{r}(\boldsymbol{x}, \boldsymbol{y})$ of order $r$ over $\mathbb{F}_{q}$ associated to $\boldsymbol{x}=$ $\left(x_{0}, \ldots, x_{n-1}\right)$ where the $x_{i}$ 's are different elements of $\mathbb{F}_{q^{m}}$ and $\boldsymbol{y}=\left(y_{0}, \ldots, y_{n-1}\right)$ where the $y_{i}$ 's are nonzero elements of $\mathbb{F}_{q^{m}}$ is defined by

$$
\mathscr{A}_{r}(\boldsymbol{x}, \boldsymbol{y})=\left\{\boldsymbol{c} \in \mathbb{F}_{q}^{n} \mid \boldsymbol{V}_{r}(\boldsymbol{x}, \boldsymbol{y}) \boldsymbol{c}^{T}=0\right\} .
$$

It should be noted that the public code in the McEliece scheme is also an alternant code. We denote here by the public code, the set of vectors of the form

$$
\left\{\boldsymbol{u} \boldsymbol{G} \mid \boldsymbol{u} \in \mathbb{F}_{q}^{k}\right\}=\left\{\boldsymbol{c S} \boldsymbol{G}_{\boldsymbol{s}} \boldsymbol{P} \mid \boldsymbol{c} \in \mathbb{F}_{q}^{k}\right\} .
$$

This is simple consequence of the fact that the set $\left\{\boldsymbol{u} \boldsymbol{S G}_{\boldsymbol{s}} \boldsymbol{P} \mid \boldsymbol{u} \in \mathbb{F}_{q}^{k}\right\}$ is obtained from the secret code $\left\{\boldsymbol{u} \boldsymbol{G}_{\boldsymbol{s}} \mid \boldsymbol{u} \in \mathbb{F}_{q}^{k}\right\}$ by permuting coordinates in it with the help of $\boldsymbol{P}$, since multiplying by an invertible matrix $\boldsymbol{S}$ of size $k \times k$ leaves the code globally invariant. The key feature of an alternant code is the following fact

Fact 1. There exists a polynomial time algorithm decoding an alternant code once a parity-check matrix $\boldsymbol{H}$ of the form $\boldsymbol{H}=\boldsymbol{V}_{r}(\boldsymbol{x}, \boldsymbol{y})$ is given.

In other words, it is possible to break the McEliece scheme, if it is possible to find $\boldsymbol{x}^{*}$ and $\boldsymbol{y}^{*}$ in $\mathbb{F}_{q^{m}}^{n}$ such that

$$
\left\{\boldsymbol{x} \boldsymbol{G} \mid \boldsymbol{x} \in \mathbb{F}_{q}^{k}\right\}=\left\{\boldsymbol{y} \in \mathbb{F}_{q}^{n} \mid \boldsymbol{V}_{r}\left(\boldsymbol{x}^{*}, \boldsymbol{y}^{*}\right) \boldsymbol{y}^{T}=0\right\}
$$


From the knowledge of this matrix $\boldsymbol{V}_{r}\left(\boldsymbol{x}^{*}, \boldsymbol{y}^{*}\right)$, it is possible to decode the public code, that is to say to recover $\boldsymbol{u}$ from $\boldsymbol{u} \boldsymbol{G}+\boldsymbol{e}$. Finding such a matrix clearly amounts to find a matrix $\boldsymbol{V}_{r}\left(\boldsymbol{x}^{*}, \boldsymbol{y}^{*}\right)$ such that $\boldsymbol{V}_{r}\left(\boldsymbol{x}^{*}, \boldsymbol{y}^{*}\right) \boldsymbol{G}^{T}=\mathbf{0}$. By bringing in $2 n$ variables $X_{0}, \ldots, X_{n-1}$ and $Y_{0}, \ldots, Y_{n-1}$ where $X_{i}$ corresponds to $x_{i}^{*}$ and $Y_{i}$ to $y_{i}^{*}$ we see that this is equivalent to solve the following system:

$$
\left\{g_{i, 0} Y_{0} X_{0}^{j}+\cdots+g_{i, n-1} Y_{n-1} X_{n-1}^{j}=0 \mid i \in\{0, \ldots, k-1\}, j \in\{0, \ldots, r-1\}\right\}
$$

where the $g_{i, j}$ 's are the entries of the known matrix $\boldsymbol{G}$ with $0 \leq i \leq k-1$ and $0 \leq j \leq r-1$.

The cryptosystems proposed in [5]27] follow the McEliece scheme [25] with the additional goal to design a public-key cryptosystem with very small key sizes. They both require to identify alternant codes having a property that allows matrices to be represented by very few rows. In the case of [5] circulant matrices are chosen whereas the scheme [27] focuses on dyadic matrices. These two families have in common the fact the matrices are completely described from the first row. The public generator matrix $\boldsymbol{G}$ in these schemes is a block matrix where each block is circulant in [5] and dyadic in [27.

We shall see that the algebraic approach previously described leads to a keyrecovery in nearly all the parameters proposed in both schemes. The crucial point that makes the attack possible is the fact that we have a system with much less unknowns than in the case of the McEliece cryptosystem. This is due to both the particular structure of the matrices and their block form that describe the public alternant codes. We finally end this section with a simple remark which explains that we can basically set one of the $Y_{i}$ 's and two values of the $X_{i}$ 's to an arbitrary value in the algebraic system (1).

Proposition 1 ([24, Chap. 10, p. 305]). Let $\left(X_{0}, \ldots, X_{n-1}\right),\left(Y_{0}, \ldots, Y_{n-1}\right)$ be a solution of (1) and $a \neq 0, b, c \neq 0$ be elements of $\mathbb{F}_{q^{m}}$. Then $\left(a X_{0}+\right.$ $\left.b, \ldots, a X_{n-1}+b\right)$ and $\left(c Y_{0}, \ldots, c Y_{n-1}\right)$ is also a solution of (1).

Solving the Algebraic System. We describe now a general technique to solve the algebraic system $\operatorname{McE}_{k, n, r}(\mathbf{X}, \mathbf{Y})$ using Gröbner bases techniques [1112 13 14]. Although the particular characteristics of the cryptosystems [5 27] studied here will further influence the shape of $\mathrm{McE}_{k, n, r}(\mathbf{X}, \mathbf{Y})$ (number of variables, number of equations, ...), we have designed a special strategy for taking advantage as much as possible of the intrinsic structure. We have made an implementation of the strategy described here. We will present the experimental results, as well as the improvements which are possible due to the quasi-cyclic and dyadic structures, in Section 4 (quasi-cyclic case) and Section 5 (dyadic case).

As a first general remark, it is readily seen that $\operatorname{McE}_{k, n, r}(\mathbf{X}, \mathbf{Y})$ is highly structured. For instance, it is very sparse as the only monomials occurring in the system are of the form $Y_{i} X_{i}^{j}$, with $0 \leq i \leq k-1$ and $0 \leq j \leq r-1$. It can also be noticed that each block of $k$ equations is bi-homogeneous, i.e. homogeneous if the variables of $\mathbf{X}$ (resp. $\mathbf{Y}$ ) are considered alone. Note that such structure already appears in the cryptanalysis of the MinRank problem [15]. 
Due to the particular structure of the system, it makes sense to design a specific strategy for solving $\operatorname{McE}_{k, n, r}(\mathbf{X}, \mathbf{Y})$. A simple way for solving this system would consist in generating the equations and try to solve it directly with a generic algorithm (for instance, the Gröbner basis algorithm available in the MAGMA computer algebra software). This approach fails for most challenges proposed in 27]. However, it is interesting to remark that this direct approach has been successful in practice for all challenges of [5]. We only mention that it takes between few minutes to 24 hours of computation using a negligible amount of memory. As a comparaison, the improved strategy that we will describe now permits to solve (almost) all the challenges of [5 27] in few seconds using also a negligible amount of memory.

The first fundamental remark is that there are $k$ linear equations in the $n$ variables of the block $\mathbf{Y}$ in $\operatorname{McE}_{k, n, r}(\mathbf{X}, \mathbf{Y})$. This implies that all the variables of the block $\mathbf{Y}$ can be expressed in terms of $d \geq n-k$ variables. From now on, we will always assume that the variables of the block $\mathbf{Y}^{\prime}$ only refer to these $d$ free variables. The first step is then to rewrite the system (1) only in function of the variables of $\mathbf{X}$ and $\mathbf{Y}^{\prime}$, i.e. the variables of $\mathbf{Y} \backslash \mathbf{Y}^{\prime}$ are substituted by linear combinations involving only variables of $\mathbf{Y}^{\prime}$. For the cryptosystems considered in this paper 527, the number of free variables $d$ in $\mathbf{Y}^{\prime}$ can be rather small (typically 1 or 2 for some challenges). Furthermore, the quasi-cyclic and dyadic structures provide additional linear equations in the variables of $\mathbf{X}$ and $\mathbf{Y}^{\prime}$ which can be also used to rewrite/clean the system. In the sequel, we will denote by $\mathrm{McE}_{k, n, r}\left(\mathbf{X}^{\prime}, \mathbf{Y}^{\prime}\right)$ the system obtained from $\operatorname{McE}_{k, n, r}(\mathbf{X}, \mathbf{Y})$ by removing all the linear equations in $\mathbf{X}$ and $\mathbf{Y}$.

The second crucial point is that as soon as the the projection of the solutions on the variables $\mathbf{Y}^{\prime}$ are known, the system (1) simplifies to:

$$
\left\{g_{i, 0}^{\prime} X_{0}^{j}+\cdots+g_{i, n-1}^{\prime} X_{n-1}^{j}=0 \mid i \in\{0, \ldots, k-1\}, j \in\{0, \ldots, r-1\}\right\} .
$$

This system is readily solved by keeping only the equations in this system which correspond to powers of the $X_{i}$ 's which are powers of two. In other words we consider only the equations of the form $g_{i, 0}^{\prime} X_{0}^{2^{j}}+\cdots+g_{i, n-1}^{\prime} X_{n-1}^{2^{j}}=0$ for $j$ in $\left\{0, \ldots,\left\lfloor\log _{2}(r-1)\right\rfloor\right\}$ and $i$ in $\{0, \ldots, k-1\}$. Hence, we obtain a quasi bi-linear system because the system is always defined over a field of characteristic two. Moreover, it has very few monomials per equation and can be easily solved in practice by computing a Gröbner basis.

The most difficult part of the computation is to find a projection of the solutions with respect to the variables of the block $\mathbf{Y}^{\prime}$. Notice that an exhaustive search on the $d$ free variables of $\mathbf{Y}^{\prime}$ leads to a practical attack for some of the challenges proposed in 527. We will present below an even more efficient strategy to recover $\mathbf{Y}^{\prime}$. More formally, let $\mathcal{I}$ be the ideal generated by $\mathrm{McE}_{k, n, r}\left(\mathbf{X}^{\prime}, \mathbf{Y}^{\prime}\right)$ and $\mathcal{V}$ be the corresponding variety i.e. the set of solutions. The goal is to compute the projection of $\mathcal{V}$, denoted by $\mathcal{V}^{\prime}$, on the variables of $\mathbf{Y}^{\prime}$. This can done by computing a Gröbner basis (w.r.t. a degree order) $G_{\text {deg }}$ of $\mathcal{I} \cap \mathbb{F}_{q^{m}}\left[\mathbf{Y}^{\prime}\right]$. This is a classical problem in computer algebra which can be solved by using standard elimination techniques (for instance see [1, Chap. 2.3, p. 69] 
or [12, Chap. 3, p. 112]. In the appendix, we briefly recall basic facts about elimination theory. In our context, we have used a slightly modified version of $\mathrm{F}_{4}\left[13\right.$ for computing a Gröbner basis $G_{\text {deg }}$ of $\mathcal{I} \cap \mathbb{F}_{q^{m}}\left[\mathbf{Y}^{\prime}\right]$. Roughly speaking, the idea is to adapt the algorithm for performing the Gröbner basis computation in $\mathbb{F}_{q^{m}}\left[\mathbf{X}^{\prime}\right]\left[\mathbf{Y}^{\prime}\right]$, i.e. the set of polynomials in $\mathbf{Y}^{\prime}$ whose coefficients are polynomials in $\mathbb{F}_{q^{m}}\left[\mathbf{X}^{\prime}\right]$. As for the usual $\mathrm{F}_{4}$, we process degree by degree. However, we consider only the degree of the polynomials w.r.t. the variables of $\mathbf{X}^{\prime}$. We stop the computation as soon as we have sufficiently many equations in $\mathbf{Y}^{\prime}$, in other words, as soon as we detect that $\mathcal{V}^{\prime}$ has a finite number of solution. Below, we describe more precisely the procedure which allows to compute a Gröbner basis $G_{\mathrm{deg}}$ of $\mathcal{I} \cap \mathbb{F}_{q^{m}}\left[\mathbf{Y}^{\prime}\right]$. As already explained, this is the most difficult part.

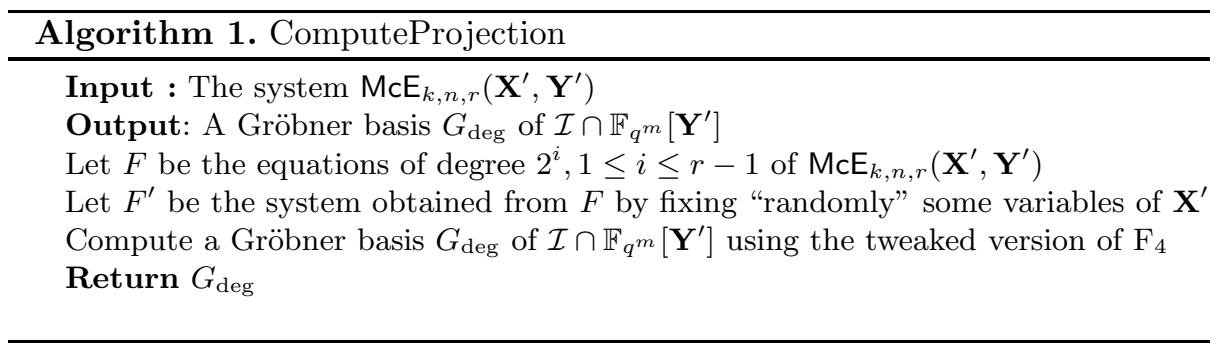

Furthermore, to be sure that the variety $\mathcal{V}^{\prime}$ associated to $\mathcal{I} \cap \mathbb{F}_{q^{m}}\left[\mathbf{Y}^{\prime}\right]$ has few solutions, we have to remove parasite solutions corresponding to $X_{i}=X_{j}$ or to $Y_{j}=0$. A classical way to do that is to introduce new variables $u_{i j}$ and $v_{i}$ and add to $\operatorname{McE}_{k, n, r}\left(\mathbf{X}^{\prime}, \mathbf{Y}^{\prime}\right)$ equations of the form:

$$
u_{i j} \cdot\left(X_{i}-X_{j}\right)+1=\text { and } v_{i} \cdot Y_{i}+1=0 .
$$

In practice, we have not added all theses equations; but only few of them namely 4 or 5 . The reason is that we do not want to add too many new variables. In addition, including few of such equations already permits to remove trivial solutions. We also have to remove some degree of freedom in the algebraic system by fixing randomly few variables of $\mathbf{X}^{\prime}$ as explained in Proposition 1. It is important to notice that since we are removing component of high dimension the new system is indeed much faster to solve.

Finally, we have not considered all the equations of $\mathrm{McE}_{k, n, r}\left(\mathbf{X}^{\prime}, \mathbf{Y}^{\prime}\right)$ to compute $G_{\text {deg }}$. This system being naturally over-defined, we can "safely" remove some equations. Typically, it makes sense to consider the smaller subset of equations such that $\mathcal{V}^{\prime}$ is zero-dimensional and for which we can efficiently compute $G_{\text {deg }}$. The variety $\mathcal{V}^{\prime}$ having few elements it is not difficult to recover this set from the knowledge of $G_{\mathrm{deg}}$. 


\section{Algebraic Cryptanalysis of the Quasi-Cyclic Variant}

The scheme presented in 5] suggests to use block matrices where each block is a circulant matrix. The public code $\mathscr{C}$ suggested in [5] is defined on a field $\mathbb{F}_{q}=\mathbb{F}_{2^{s}}$ which is considered as a subfield of $\mathbb{F}_{q^{m}}$ for a certain integer $m$. Let $\alpha$ be a primitive element of $\mathbb{F}_{q^{m}}$. Let $\ell$ and $N_{0}$ be such that $q^{m}-1=N_{0} \ell$ and let $\beta$ be an element of $\mathbb{F}_{q^{m}}$ of order $\ell$. Although this is not explicitly stated in [5], it is readily checked that $\mathscr{C}$ is defined from an $r \times n$ parity-check matrix $\boldsymbol{H}$ over $\mathbb{F}_{q^{m}}$ which is the juxtaposition of $n_{0}\left(n=\ell n_{0}\right)$ matrices $\boldsymbol{H}^{(0)}, \cdots, \boldsymbol{H}^{\left(n_{0}-1\right)}$ of size $r \times \ell$ Each $\boldsymbol{H}^{(b)}=\left(h_{i, j}^{(b)}\right)$ with $0 \leq b \leq n_{0}-1,0 \leq i \leq r-1$ and $0 \leq j \leq \ell-1$ is given by

$$
h_{i, j}^{(b)}=\gamma_{b} \beta^{\left(d_{b}+j\right) e}\left(\alpha^{w_{b}} \beta^{d_{b}+j}\right)^{i}
$$

where $\gamma_{b}$ is a nonzero element of $\mathbb{F}_{q^{m}}, d_{b}$ is an integer of $\{0, \ldots, \ell-1\}, e$ is an integer of $\{0, \ldots, \ell-1\}$ and the $w_{b}$ 's are distinct integers of $\left\{0, \ldots, N_{0}-1\right\}$. From this, it is now clear that $\mathscr{C}$ is an alternant code $\mathscr{A}_{r}(\boldsymbol{x}, \boldsymbol{y})$ of order $r$ associated to $\boldsymbol{x}=\left(x_{0}, \ldots, x_{n-1}\right)$ and $\boldsymbol{y}=\left(y_{0}, \ldots, y_{n-1}\right)$ which satisfy for any $j$ in $\{0, \ldots, \ell-1\}$

$$
\begin{aligned}
& x_{b \ell+j}=\alpha^{w_{b}} \beta^{d_{b}+j} \\
& y_{b \ell+j}=\gamma_{b} \beta^{\left(d_{b}+j\right) e},
\end{aligned}
$$

It can be checked (see [5]) that $\mathscr{C}$ has a public generating matrix $\boldsymbol{G}$ which is block circulant of size $k \times n$ with $k$ of the form $k=k_{0} \ell$ for some integer $k_{0}$ (recall that $k \geq n-r m)$.

We present now an algebraic attack against the quasi-cyclic variant proposed in 5] that recovers $\boldsymbol{x}$ and $\boldsymbol{y}$ by setting up an algebraic system of the form $\mathrm{McE}_{k, n, r}(\mathbf{X}, \mathbf{Y})$ from the equation $\boldsymbol{H} \boldsymbol{G}^{T}=0$. This would also give a system with $2 n$ unknowns. We can obtain a huge reduction of the number of unknowns by using Equations (7) and (8) which induce some linear relations between the $x_{i}$ 's and the $y_{i}$ 's. From these two equations we deduce that

$$
\begin{aligned}
& x_{b \ell+j}=x_{b \ell} \beta^{j} \\
& y_{b \ell+j}=y_{b \ell} \beta^{j e},
\end{aligned}
$$

for any $j$ in $\{0, \ldots, \ell-1\}$ and $j$ in $\left\{0, \ldots, n_{0}-1\right\}$. Furthermore, since in the cases considered in [5], $e$ is small because it lies in the range $\{0, \ldots, \ell-1\}$ and $\ell$ is less than 100 , we may assume that:

Assumption 2. The secret integer e such that $0 \leq e \leq \ell-1$ is known.

This enables to simplify the description of the system $\operatorname{McE}_{k, n, r}(\mathbf{X}, \mathbf{Y})$. By setting up the unknown $X_{b}$ for $x_{b \ell}$ and the unknown $Y_{b}$ for $y_{b \ell}$ we obtain the following algebraic system.

Proposition 2. Let $\boldsymbol{G}=\left(g_{i, j}\right)$ be the $k \times n$ public generator matrix with $k=k_{0} \ell$ and $n=n_{0} \ell$. For any $0 \leq w \leq r-1$ and any $0 \leq i \leq k-1$, the unknowns $X_{0}, \ldots, X_{n_{0}-1}$ and $Y_{0}, \ldots, Y_{n_{0}-1}$ should satisfy: 


$$
\sum_{b=0}^{n_{0}-1} g_{i, b, w}^{\prime} Y_{b} X_{b}^{w}=0 \quad \text { where } g_{i, b, w}^{\prime} \stackrel{\text { def }}{=} \sum_{j=0}^{\ell-1} g_{i, b \ell+j} \beta^{j(e+w)} .
$$

Proof. We observe that

$$
\begin{aligned}
\sum_{j=0}^{n-1} g_{i, j} y_{j} x_{j}^{w} & =\sum_{b=0}^{n_{0}-1} \sum_{j=0}^{\ell-1} g_{i, b \ell+j} y_{b \ell+j} x_{b \ell+j}^{w}=\sum_{b=0}^{n_{0}-1} \sum_{j=0}^{\ell-1} g_{i, b \ell+j} y_{b \ell} x_{b \ell}^{w} \beta^{j e} \beta^{j w} \\
& =\sum_{b=0}^{n_{0}-1} y_{b \ell} x_{b \ell}^{w}\left(\sum_{j=0}^{\ell-1} g_{i, b \ell+j} \beta^{j e} \beta^{j w}\right)
\end{aligned}
$$

By setting $X_{b}$ for $x_{b \ell}$ and $Y_{b}$ for $y_{b \ell}$ we obtain the aforementioned system.

Theoretically by Proposition [1, we would be able to fix two variables, say $X_{0}$ and $X_{1}$, and one variable $Y_{j}$, for instance $Y_{0}$, to arbitrary values as long as $X_{0} \neq X_{1}$ and $Y_{0} \neq 0$. However, if we do it, we then lose the linear relations between the $x_{i}$ 's given in (9). Therefore we can only fix one $X_{i}$ and one $Y_{i}$ as stated in Lemma 1 that is straightforward to prove.

Lemma 1. Let $\left(X_{0}, \ldots, X_{n_{0}-1}\right),\left(Y_{0}, \ldots, Y_{n_{0}-1}\right)$ be a solution of (11). Then $\left(a X_{0}, \ldots, a X_{n_{0}-1}\right)$ and $\left(c Y_{0}, \ldots, c Y_{n_{0}-1}\right)$ is also a solution of (11) for any $a \neq 0$ and $c \neq 0$ of $\mathbb{F}_{q^{m}}$.

Hence, the total number of unknowns is $2\left(n_{0}-1\right)$. Furthermore there are many redundant equations in Proposition 2, This comes from the block circulant form of $\boldsymbol{G}$. From this form we know that $g_{i \ell+u, b \ell+j}=g_{i \ell, b \ell+((j-u) \bmod \ell)}$ for all $u$ in $\{0, \ldots, \ell-1\}$ and $i$ in $\left\{0, \ldots, k_{0}-1\right\}$. We also have:

$$
\begin{aligned}
g_{i \ell+u, b, w}^{\prime} & =\sum_{j=0}^{\ell-1} g_{i \ell+u, b \ell+j} \beta^{j(e+w)}=\sum_{j=0}^{\ell-1} g_{i \ell, b \ell+((j-u) \bmod \ell)} \beta^{j(e+w)} \\
& =\sum_{j=0}^{\ell-1} g_{i \ell, b \ell+j} \beta^{j(e+w)} \beta^{u(e+w)}=g_{i \ell, b, w}^{\prime} \beta^{u(e+w)}
\end{aligned}
$$

We used the fact $\beta^{\ell(e+w)}=1$. So for a given $i$, when $u$ describes $\{0, \ldots, \ell-1\}$, the equations $\sum_{b=0}^{n_{0}-1} g_{i \ell+u, b, w}^{\prime} Y_{b} X_{b}^{w}=0$ are all equivalent. This means that instead of having $r k$ equations we have only $\frac{r k}{\ell}=k_{0} r$ algebraic equations.

Proposition 3. The system (11) has $\left(n_{0}-1\right)$ unknowns $Y_{i}$ and $\left(n_{0}-1\right)$ unknowns $X_{i}$. Furthermore, it has $k_{0}$ linear equations involving only the $Y_{i}$ 's and $(r-1) k / \ell=(r-1) k_{0}$ polynomial equations involving the unknowns $Y_{i} X_{i}^{w}$ with $w>1$.

From now on, we will always assume that redundant equations have been removed and the variables $X_{0}$ and $Y_{0}$ are fixed. Finally, note that there are $d \stackrel{\text { def }}{=} n_{0}-1-k_{0}$ free variables for the $Y_{i}$ 's. 
Table 1. Cryptanalysis results for $[\underline{5}](m=2)$

\begin{tabular}{|c|c|c|c|c|c|c|c|}
\hline Challenge & $q$ & $\bar{\ell}$ & \begin{tabular}{|l|l|}
$n_{0}$ & $d$ \\
\end{tabular} & \begin{tabular}{|l|l} 
Security & 5 \\
\end{tabular} & Unknowns & Equations & Time (Operations, Memory)] \\
\hline 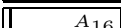 & $2^{8}$ & 51 & \begin{tabular}{l|l}
9 & 3 \\
\end{tabular} & 80 & 16 & 510 & $0.06 \mathrm{sec}\left(2^{18.9}\right.$ op, $\left.115 \mathrm{Meg}\right)$ \\
\hline$B_{16}$ & $2^{8}$ & 51 & 103 & 90 & 18 & 612 & $0.03 \sec \left(2^{17.1} \mathrm{op}, 116 \mathrm{Meg}\right)$ \\
\hline$C_{16}$ & $2^{8}$ & 51 & 123 & 100 & 22 & 816 & $0.05 \mathrm{sec}\left(2^{16.2} \mathrm{op}, 116 \mathrm{Meg}\right)$ \\
\hline$D_{16}$ & $2^{8}$ & 51 & \begin{tabular}{|l|l|}
15 & 4 \\
\end{tabular} & 120 & 28 & 1275 & $0.02 \sec \left(2^{14.7} \mathrm{op}, 113 \mathrm{Meg}\right)$ \\
\hline 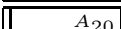 & $22^{10}$ & 75 & $\begin{array}{ll}6 & 2\end{array}$ & 80 & 10 & 337 & $0.05 \mathrm{sec}\left(2^{15.8} \mathrm{op}, 115 \mathrm{Meg}\right)$ \\
\hline$B_{20}$ & 210 & 93 & 62 & 90 & 10 & 418 & $0.05 \sec \left(2^{17.1} \mathrm{op}, 115 \mathrm{Meg}\right)$ \\
\hline$C_{20}$ & $2^{10}$ & 93 & $\begin{array}{lll}8 & 2\end{array}$ & 110 & 14 & 697 & $0.02 \mathrm{sec}\left(2^{14.5} \mathrm{op}, 115 \mathrm{Meg}\right)$ \\
\hline$\overline{\mathrm{QC}_{600}}$ & $2^{8}$ & 255 & \begin{tabular}{|l|l|}
15 & 3 \\
\end{tabular} & 600 & 28 & 6820 & $0.08 \mathrm{sec}\left(2^{16.6} \mathrm{op}, 116 \mathrm{Meg}\right)$ \\
\hline
\end{tabular}

We now present experimental results obtained when solving the system described in (11) using the strategy described in Section 3. The experimental results have been obtained with several Xeon bi-processor $3.2 \mathrm{Ghz}$, with $16 \mathrm{~Gb}$ of Ram. The instances of our problem have been generated using the MAGMA software. We used the MAgma version 2.15 for our computations. The $\mathrm{F}_{5}$ [14] algorithm has been implemented in $\mathrm{C}$ language in the FGb software. We used this implementation for computing the first Gröbner basis (i.e. which is used in Algorithm 1). All the other computations are performed under MAGMA including factorizing some univariate polynomials and computing Gröbner bases using the $\mathrm{F}_{4}$ [13 algorithm. The most important observation is that we have been able to solve all the challenges of [5] in a negligible time because the dimension $d=n_{0}-1-k_{0}$ of the vector space solution for the $Y_{i}$ 's is very small. We also proposed a challenge $\mathrm{QC}_{600}$ for showing the behaviour of our attack for high security levels.

\section{Algebraic Cryptanalysis of the Dyadic Variant}

The cryptosystem presented in 27] considers particular alternant codes called quasi-dyadic Goppa codes. Goppa codes form an important subclass of alternant codes. Goppa codes are defined by means of a polynomial $G(X)$ of degree $\ell$ with coefficients in $\mathbb{F}_{q^{m}}$ and for which the sequence $\boldsymbol{x}$ is assumed not to contain any root of $G(X)$. The alternant code defined by the parity-check matrix $\boldsymbol{V}_{\ell}(\boldsymbol{x}, \boldsymbol{y})$ with $y_{i}=G\left(x_{i}\right)^{-1}$ is called a Goppa code over $\mathbb{F}_{q}$ and is denoted by $\mathscr{G}(\boldsymbol{x}, G)$. A detailed description of the key generation is given in Appendix B. We only provide important facts that are useful for recovering the private key. We first state an important result that shows that $\boldsymbol{G}$ defines actually an alternant code. The proof is given in Appendix C. The last important fact to know about $\boldsymbol{G}$ is that it is a $k \times n$ matrix over $\mathbb{F}_{q}$ such that $n=n_{0} \ell$ and $k \geq n-m \ell$ where $n_{0}, \ell$ are given integers.

Proposition 4. The code defined by the public generator matrix $\boldsymbol{G}$ is an alternant code $\mathscr{A}_{\ell}(\boldsymbol{x}, \boldsymbol{y})$ where for any $0 \leq j \leq n_{0}-1$ and $0 \leq i, i^{\prime} \leq \ell-1$, we have the following equations: 


$$
\left\{\begin{aligned}
y_{j \ell+i} & =y_{j \ell} \\
x_{j \ell+i}+x_{j \ell} & =x_{i}+x_{0} \\
x_{j \ell+\left(i \oplus i^{\prime}\right)} & =x_{j \ell+i}+x_{j \ell+i^{\prime}}+x_{j \ell}
\end{aligned}\right.
$$

where $\oplus$ is the bitwise exclusive-or on the binary representation of the indices.

The cryptanalysis of the system consists in defining $n_{0}$ unknowns $Y_{0}, \ldots, Y_{n_{0}-1}$ that play the role of the $y_{j}$ 's and $n$ unknowns $X_{0}, \ldots, X_{n}$ that represent the $x_{j}$ 's. We know specify the system of equations that we obtain directly from Proposition 4 .

Proposition 5. For any $w, j, i$ and $i^{\prime}$ such that $0 \leq w \leq \ell-1,0 \leq j \leq n_{0}-1$ and $1 \leq i, i^{\prime} \leq \ell-1$, we have:

$$
\left\{\begin{array}{r}
\sum_{j=0}^{n_{0}-1} Y_{j} \sum_{l=0}^{\ell-1} g_{i, j \ell+l} X_{j \ell+l}^{w}=0 \\
X_{j \ell+i}+X_{j \ell}+X_{i}+X_{0}=0 \\
X_{j \ell+\left(i \oplus i^{\prime}\right)}+X_{j \ell+i}+X_{j \ell+i^{\prime}}+X_{j \ell}=0
\end{array}\right.
$$

It is possible to simplify System (13) by observing, thanks to the third equation, that actually many variables $X_{i}$ 's can be expressed in function of some few variables, namely $X_{2^{j}}$ with $0 \leq j \leq \log _{2}(\ell-1)$ and $X_{b}$ with $0 \leq b \leq n_{0}-1$.

Corollary 1. For any $1 \leq i \leq \ell-1$, if we write the binary decomposition of $i=\sum_{j=0}^{\log _{2}(\ell-1)} \eta_{j} 2^{j}$ then the following equation holds:

$$
X_{i}=X_{0}+\sum_{j=0}^{\log _{2}(\ell-1)} \eta_{j}\left(X_{2^{j}}+X_{0}\right) .
$$

We are also able to provide the exact number of unknowns we can fix to arbitrary values.

Lemma 2. Let $\left(X_{0}, \ldots, X_{n-1}\right),\left(Y_{0}, \ldots, Y_{n-1}\right)$ be a solution of (13) and $a \neq 0$, $b, c \neq 0$ be elements of $\mathbb{F}_{q^{m}}$. Then $\left(a X_{0}+b, \ldots, a X_{n-1}+b\right)$ and $\left(c Y_{0}, \ldots, c Y_{n-1}\right)$ is also a solution of (13).

Proof. The only fact to prove is that $\left(X_{0}+b, \ldots, X_{n-1}+b\right)$ is also a solution of the last two equations in (13). It is readily checked since $\mathbb{F}_{q^{m}}$ is of charateristic two.

We can now completely give the effective number of equations after elimination of redundant equations.

Proposition 6. The system (13) has $n_{0}-1$ unknowns $Y_{i}$ and $n_{0}-2+\log _{2}(\ell)$ unknowns $X_{i}$. Furthermore, it has $n_{0}-m$ linear equations involving only the $Y_{i}$ 's, and $(\ell-1) \ell\left(n_{0}-m\right)$ polynomial equations involving the unknowns $Y_{i} X_{i}^{w}$ with $w>1$. 
Proof. The number of variables $Y_{j}$ is $\left(n_{0}-1\right)$ since we can choose $Y_{0}=1$. As for the variables $X_{j}$, we observe that they can all be expressed only in function of $X_{2^{j}}$ and $X_{i \ell}$ with $0 \leq j \leq \log _{2}(\ell-1)$ and $0 \leq i \leq n_{0}-1$. So the number of unknowns $X_{j}$ is $\log _{2}(\ell-1)+1+n_{0}-2$ since we can fix two different arbitrary values for two variables, say $X_{0}$ and $X_{\ell}$ (Lemma 2). Using the fact that $\log _{2}(\ell-1)=\log _{2}(\ell)-1$ since $\ell$ is a power of 2 , we get the claimed number of unknowns. Furthermore, because of the dyadicity of $\boldsymbol{G}$, the equations obtained with $w=0$ are identical when $\boldsymbol{g}$ describes all the rows of a dyadic block of $\boldsymbol{G}$. This does not appear when $w>1$. So we have $k / \ell=n_{0}-m$ linear equations that involve the $Y_{i}$ 's and $(\ell-1) k=(\ell-1) \ell\left(n_{0}-m\right)$ polynomial equations that contain variables of the form $Y_{i} X_{i}^{w}$ where $w>1$.

We now present in Table 2 the experimental results we obtained when we solve the system described in (13) using the strategy described in Section 3. As previously, the experimental results have been obtained with several Xeon bi-processor $3.2 \mathrm{Ghz}$, with $16 \mathrm{~Gb}$ of Ram. The instances of our problem have been generated using the Magma software. We used the MAGma version 2.15 for our computations. The $\mathrm{F}_{5}[14$ algorithm has been implemented in $\mathrm{C}$ language in the $\mathrm{FGb}$ software. We used this implementation for computing the first Gröbner basis (i.e. which is used in Algorithm 1). All the other computations are performed under MAGMA including factorizing some univariate polynomials and computing Gröbner bases using the $\mathrm{F}_{4}$ algorithm 13 . Table 2 also shows the impact of the degree extension $m$. Indeed, computation times indicate that the solutions are easy to find if $m$ is small. This phenomenon is directly related to the size of the solution space of the variables $Y_{i}$. We have seen in Section 3 that such variables satisfy a system of linear equations. From Proposition 6 , the number of linear equations is $k_{0}=n_{0}-m$ whereas the number of unknowns $Y_{i}$ is $n_{0}-1$. Thus the dimension of the vector space solution for the $Y_{i}$ 's is $m-1$. We also give in Table 2 new parameters Dyadic $_{256}$ and Dyadic $_{512}$ ) that are generated by "extrapolating" challenges given in [27. We observe that this solution space is manageable in practise as long as $m<16$ because we did not succeed to find an efficient way to solve the challenges of [27] when $m=16$.

Table 2. Cryptanalysis results for 27 .

\begin{tabular}{|c|c|c|c|c|c|c|c|c|}
\hline Challenge & $\bar{q}$ & $m$ & $\ell$ & $n_{0}$ & Security & Unknowns & Equations & $\overline{\bar{T} \text { Time (Operations, Memory) }}$ \\
\hline Table 2 & $2^{2}$ & 8 & 64 & 56 & 128 & 115 & 193,584 & \multirow{3}{*}{$\begin{array}{r}1,776.3 \mathrm{sec}\left(2^{34.2} \text { op, } 360 \mathrm{Meg}\right) \\
0.50 \mathrm{sec}\left(2^{22.1} \text { op, } 118 \mathrm{Meg}\right) \\
0.03 \mathrm{sec}\left(2^{16.7} \text { op, } 35 \mathrm{Meg}\right) \\
\end{array}$} \\
\hline Table 2 & $2^{4}$ & 4 & 64 & 32 & 128 & 67 & 112,924 & \\
\hline Table 2 & $2^{8}$ & 2 & 64 & 12 & 128 & 27 & 40,330 & \\
\hline Table 3 & $2^{8}$ & 2 & 64 & 10 & 102 & 23 & 32,264 & \multirow{3}{*}{$\begin{array}{l}0.03 \text { sec }\left(2^{15.9} \text { op, } 113 \mathrm{Meg}\right) \\
0.02 \sec \left(2^{15.4} \text { op, } 113 \mathrm{Meg}\right) \\
0.11 \text { sec }\left(2^{19.2} \text { op, } 113 \mathrm{Meg}\right) \\
\end{array}$} \\
\hline Table 3 & $2^{8}$ & 2 & 128 & 6 & 136 & 16 & 65,028 & \\
\hline Table 3 & $2^{8}$ & 2 & 256 & 4 & 168 & 13 & 130,562 & \\
\hline Table 5 & $2^{8}$ & 2 & 128 & 4 & 80 & 12 & 32,514 & \multirow{5}{*}{ 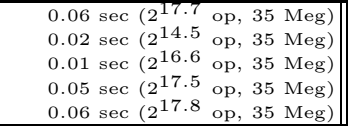 } \\
\hline Table 5 & $2^{8}$ & 2 & 128 & 5 & 112 & 14 & 48,771 & \\
\hline Table 5 & $2^{8}$ & 2 & 128 & 6 & 128 & 16 & 65,028 & \\
\hline Table 5 & $2^{8}$ & 2 & 256 & 5 & 192 & 15 & 195,843 & \\
\hline Table 5 & $2^{8}$ & 2 & 256 & 6 & 256 & 17 & 261,124 & \\
\hline $\operatorname{yadic}_{256}$ & $2^{4}$ & 4 & 128 & 32 & 256 & 68 & 455,196 & \multirow{2}{*}{$\begin{array}{l}7.1 \mathrm{sec}\left(2^{26.1} \text { op, } 131 \mathrm{Meg}\right) \\
0.15 \mathrm{sec}\left(2^{19.7} \text { op, } 38 \mathrm{Meg}\right)\end{array}$} \\
\hline yadic & $2^{8}$ & 2 & 512 & 6 & 512 & 18 & $1,046,532$ & \\
\hline
\end{tabular}




\section{Conclusion}

We described in this paper a new algebraic approach to assess the security of the McEliece cryptosystem. We showed that the private key of this scheme is a solution of a very structured system of bi-homogeneous polynomial equations in two sets of unknowns $Y_{i}$ and $X_{i}$. The solutions belong to a finite field $\mathbb{F}_{q^{m}}$ whereas the coefficients of the system are in $\mathbb{F}_{q}$ for some known integers $m$ and $q$. This system comes from the particular structure of alternant codes used in McEliece. Indeed, the Goppa codes as proposed in 25 form a subfamily of alternant codes. Furthermore, the system is composed of two parts of equations: one part that consists of linear equations that involve only the unknowns $Y_{i}$ and a second part where the equations involve terms of the form $Y_{i} X_{i}^{j}$.

We applied this approach to two new cryptosystems [27/5] that are variants of the McEliece scheme. Both aim at reducing the public keys by using very structured block matrices (cyclic matrices in [5] and dyadic matrices in [27]). We show that our new cryptanalytic point of view is very efficient for all the parameters proposed in [5]. An implementation in MAGMA validates our attack and shows that the private key can be found in a negligible time. For the scheme [27, we are also able to fully recover the private key in almost all cases. An implementation in MAGMA shows that this can be done in time comparable to [5] as long as the dimension $m$ of the solution vector space of the $Y_{i}$ 's is small.

Thanks to a very recent development [16] on the solving of bihomogeneous bilinear systems, it is very likely that the solving technique presented here can be replaced by a new version of $\mathrm{F}_{5}$ dedicated to bi-linear systems. In our case, we can obtain a (quasi) bilinear system when we consider the equations involving terms of the form $Y_{i} X_{i}^{2^{j}}$. Moreover, this will permit to precisely estimate the complexity of the attack presented in this paper and will provide a concrete criteria to evaluate the security of future compact McEliece's variants.

Finally, it would be interesting to study if this algebraic approach followed here can be improved in order to mount an attack on the original McEliece cryptosystem. In this case however, there are much more unknowns than in the cases considered here and there is much more freedom left on the $Y_{i}$ 's by looking at the linear equations involving only the $Y_{i}$ 's.

\section{References}

1. Adams, W., Loustaunau, P.: An Introduction to Gröbner Bases. American Mathematical Society, Providence (July 1994)

2. Avanzi, R.: Lightweight asymmetric cryptography and alternatives to rsa, ecrypt european network of excellence in cryptology (2005), http://www .ecrypt.eu.org/ecrypt1/documents/D.AZTEC.2-1.2.pdf

3. Baldi, M., Bodrato, M., Chiaraluce, G.F.: A new analysis of the McEliece cryptosystem based on QC-LDPC codes. In: Ostrovsky, R., De Prisco, R., Visconti, I. (eds.) SCN 2008. LNCS, vol. 5229, pp. 246-262. Springer, Heidelberg (2008)

4. Baldi, M., Chiaraluce, G.F.: Cryptanalysis of a new instance of McEliece cryptosystem based on QC-LDPC codes. In: IEEE International Symposium on Information Theory, Nice, France, March 2007, pp. 2591-2595 (2007) 
5. Berger, T.P., Cayrel, P.L., Gaborit, P., Otmani, A.: Reducing key length of the McEliece cryptosystem. In: Preneel, B. (ed.) AFRICACRYPT 2009. LNCS, vol. 5580, pp. 77-97. Springer, Heidelberg (2009)

6. Berger, T.P., Loidreau, P.: How to mask the structure of codes for a cryptographic use. Designs Codes and Cryptography 35(1), 63-79 (2005)

7. Berger, T.P., Loidreau, P.: Designing an efficient and secure public-key cryptosystem based on reducible rank codes. In: Canteaut, A., Viswanathan, K. (eds.) INDOCRYPT 2004. LNCS, vol. 3348, pp. 218-229. Springer, Heidelberg (2004)

8. Bernstein, D.J., Lange, T., Peters, C.: Attacking and defending the McEliece cryptosystem. In: Buchmann, J., Ding, J. (eds.) PQCrypto 2008. LNCS, vol. 5299, pp. 31-46. Springer, Heidelberg (2008)

9. Bernstein, D.J., Lange, T., Peters, C., van Tilborg, H.: Explicit bounds for generic decoding algorithms for code-based cryptography. In: Pre-proceedings of WCC 2009, pp. 168-180 (2009)

10. Biswas, B., Sendrier, N.: McEliece cryptosystem implementation: Theory and practice. In: Buchmann, J., Ding, J. (eds.) PQCrypto 2008. LNCS, vol. 5299, pp. 47-62. Springer, Heidelberg (2008)

11. Buchberger, B.: Ein Algorithmus zum Auffinden der Basiselemente des Restklassenringes nach einem nulldimensionalen Polynomideal. PhD thesis, Innsbruck (1965)

12. Cox, D.A., Little, J.B., O'Shea, D.: Ideals, Varieties, and algorithms: an Introduction to Computational Algebraic Geometry and Commutative Algebra. Undergraduate Texts in Mathematics. Springer, New York (2001)

13. Faugère, J.-C.: A new efficient algorithm for computing gröbner bases (f4). Journal of Pure and Applied Algebra 139(1-3), 61-88 (1999)

14. Faugère, J.-C.: A new efficient algorithm for computing gröbner bases without reduction to zero: F5. In: ISSAC 2002, pp. 75-83. ACM press, New York (2002)

15. Faugère, J.-C., Levy-dit Vehel, F., Perret, L.: Cryptanalysis of minrank. In: Wagner, D. (ed.) CRYPTO 2008. LNCS, vol. 5157, pp. 280-296. Springer, Heidelberg (2008)

16. Faugère, J.-C., El Din, M.S., Spaenlehauer, P.-J.: Gröbner bases of bihomogeneous ideals generated by polynomials of bidegree $(1,1)$ : Algorithms and complexity. CoRR, abs/1001.4004 (2010)

17. Faugère, J.-C., Gianni, P.M., Lazard, D., Mora, T.: Efficient computation of zerodimensional gröbner bases by change of ordering. J. Symb. Comput. 16(4), 329-344 (1993)

18. Faure, C., Minder, L.: Cryptanalysis of the McEliece cryptosystem over hyperelliptic curves. In: Proceedings of the eleventh International Workshop on Algebraic and Combinatorial Coding Theory, Pamporovo, Bulgaria, June 2008, pp. 99-107 (2008)

19. Finiasz, M., Sendrier, N.: Security bounds for the design of code-based crypto systems. In: Matsui, M. (ed.) ASIACRYPT 2009. LNCS, vol. 5912, pp. 88-105. Springer, Heidelberg (2009)

20. Gabidulin, E., Paramonov, A.V., Tretjakov, O.V.: Ideals over a non-commutative ring and their applications to cryptography. In: Davies, D.W. (ed.) EUROCRYPT 1991. LNCS, vol. 547, pp. 482-489. Springer, Heidelberg (1991)

21. Gaborit, P.: Shorter keys for code based cryptography. In: Ytrehus, Ø. (ed.) WCC 2005. LNCS, vol. 3969, pp. 81-91. Springer, Heidelberg (2006)

22. Gibson, J.K.: Severely denting the Gabidulin version of the McEliece public key cryptosystem. Design Codes and Cryptography 6(1), 37-45 (1995) 
23. Janwa, H., Moreno, O.: McEliece public key cryptosystems using algebraicgeometric codes. Designs Codes and Cryptography 8(3), 293-307 (1996)

24. MacWilliams, F.J., Sloane, N.J.A.: The Theory of Error-Correcting Codes, 5th edn. North-Holland, Amsterdam (1986)

25. McEliece, R.J.: A Public-Key System Based on Algebraic Coding Theory, pp. 114116. Jet Propulsion Lab. (1978); DSN Progress Report 44

26. Minder, L., Shokrollahi, A.: Cryptanalysis of the Sidelnikov cryptosystem. In: Naor, M. (ed.) EUROCRYPT 2007. LNCS, vol. 4515, pp. 347-360. Springer, Heidelberg (2007)

27. Misoczki, R., Barreto, P.S.L.M.: Compact McEliece keys from Goppa codes. In: Jacobson Jr., M.J., Rijmen, V., Safavi-Naini, R. (eds.) SAC 2009. LNCS, vol. 5867, pp. 376-392. Springer, Heidelberg (2009)

28. Niederreiter, H.: A public-key cryptosystem based on shift register sequences. In: Pichler, F. (ed.) EUROCRYPT 1985. LNCS, vol. 219, pp. 35-39. Springer, Heidelberg (1985)

29. Otmani, A., Tillich, J.P., Dallot, L.: Cryptanalysis of McEliece cryptosystem based on quasi-cyclic ldpc codes. In: Proceedings of First International Conference on Symbolic Computation and Cryptography, Beijing, China, April 28-30, pp. 69-81. LMIB Beihang University (2008)

30. Overbeck, R.: Structural attacks for public key cryptosystems based on Gabidulin codes. J. Cryptology 21(2), 280-301 (2008)

31. Sidelnikov, V.M.: A public-key cryptosytem based on Reed-Muller codes. Discrete Mathematics and Applications 4(3), 191-207 (1994)

32. Sidelnikov, V.M., Shestakov, S.O.: On the insecurity of cryptosystems based on generalized Reed-Solomon codes. Discrete Mathematics and Applications 1(4), 439 444 (1992)

33. Stern, J.: A method for finding codewords of small weight. In: Wolfmann, J., Cohen, G. (eds.) Coding Theory 1988. LNCS, vol. 388, pp. 106-113. Springer, Heidelberg (1988)

34. Gauthier Umana, V., Leander, G.: Practical key recovery attacks on two McEliece variants (2009), http://eprint.iacr.org/2009/509

35. Wieschebrink, C.: Cryptanalysis of the Niederreiter public key scheme based on GRS subcodes. eprint 452 (2009), http://eprint.iacr.org/2009/452.pdf 


\section{A Gröbner Basics}

The classical approach for computing a Gröbner basis of $\mathcal{I} \cap \mathbb{F}_{q^{m}}\left[\mathbf{Y}^{\prime}\right]$ can be described as follows. A reader already familiar with polynomial system solving can skip this part. We have to choose a suitable ordering on the monomials (for a definition of such orders, see for instance [12, Chap. 2, p. 52]). In particular, we have to select an elimination ordering ([1, Chap. 2.3, p. 69]) on the blocks $\mathbf{X}^{\prime}, \mathbf{Y}^{\prime}$ such that the variables occurring in $\mathbf{X}$ are greater that those of $\mathbf{Y}^{\prime}$ (denoted by $\mathbf{X}^{\prime}>>\mathbf{Y}^{\prime}$ ). According to [1, Theo. 2.3.4, Chap. 2.3, p. 69], this elimination ordering will permit to compute a Gröbner basis $G_{\mathrm{deg}}$ of $\mathcal{I} \cap \mathbb{F}_{q^{m}}\left[\mathbf{Y}^{\prime}\right]$ with respect to a degree order on the variables of $\mathbf{Y}^{\prime}$ (i.e. this is the order induced when "removing" the variables of the block $\mathbf{X}^{\prime}$ in the elimination ordering). In theory, to compute the variety $\mathcal{V}^{\prime}$ associated to $\mathcal{I} \cap \mathbb{F}_{q^{m}}\left[\mathbf{Y}^{\prime}\right]$, we have to perform a change of ordering on $G_{\text {deg }}$ to compute Gröbner basis $G_{\text {lex }}$ of $\mathcal{I} \cap \mathbb{F}_{q^{m}}\left[\mathbf{Y}^{\prime}\right]$. If we assume that $\mathcal{V}^{\prime}$ is zero-dimensional (i.e. has a finite number of solutions so that $\left.\# \mathcal{V}^{\prime}<\infty\right)$, then an efficient tool to perform the change of ordering is the FGLM algorithm [17. The complexity of computing $G_{\text {lex }}$ from $G_{\text {deg }}$ with FGLM is polynomial in the size of $\mathcal{V}^{\prime}$, i.e. $\mathcal{O}\left(\left(\# \mathcal{V}^{\prime}\right)^{3}\right)$. In our case, the size of $\mathcal{V}^{\prime}$ is very small $(<10)$.

We have used a slightly modified version of $\mathrm{F}_{4}$ [13] for computing a Gröbner basis $G_{\text {deg }}$ of $\mathcal{I} \cap \mathbb{F}_{q^{m}}\left[\mathbf{Y}^{\prime}\right]$. The idea is to adapt the algorithm for performing the Gröbner basis computation in $\mathbb{F}_{q^{m}}\left[\mathbf{X}^{\prime}\right]\left[\mathbf{Y}^{\prime}\right]$, i.e. the set of polynomials in $\mathbf{Y}^{\prime}$ whose coefficients are polynomials in $\mathbb{F}_{q^{m}}\left[\mathbf{X}^{\prime}\right]$. As for the usual $\mathrm{F}_{4}$, we process degree by degree. However, we consider only the degree of the polynomials w.r.t. the variables of $\mathbf{X}^{\prime}$. We stop the computation as soon as we have sufficiently many equations in $\mathbf{Y}^{\prime}$ (for instance, as soon as we detect that $\mathcal{V}^{\prime}$ has a finite number of solution, i.e. of dimension zero ). The modified version is defined below.

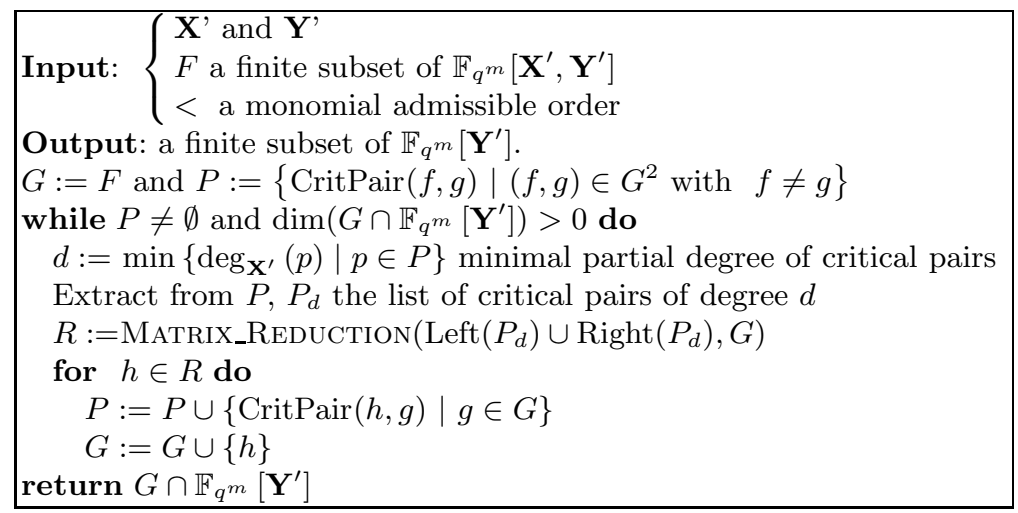

Fig. 1. Algorithm $F_{4}$ (modified version) 
For the definition of Matrix_Reduction, and CritPair, we refer to [13]. Briefly, the first function performs the usual polynomial reduction of Buchberger's algorithm [12] using linear algebra. The second function selects critical pairs with respect to a defined strategy.

\section{B Description of the Variant Based on Dyadic Goppa Codes}

The cryptosystem presented in [27] considers particular alternant codes called quasi-dyadic Goppa codes. Goppa codes form an important subclass of alternant codes. Goppa codes are defined by means of a polynomial $G(X)$ of degree $\ell$ with coefficients in $\mathbb{F}_{q^{m}}$ and for which the sequence $\boldsymbol{x}$ is assumed not to contain any root of $G(X)$. The alternant code defined by the parity-check matrix $\boldsymbol{V}_{\ell}(\boldsymbol{x}, \boldsymbol{y})$ with $y_{i}=G\left(x_{i}\right)^{-1}$ is called a Goppa code over $\mathbb{F}_{q}$ and is denoted by $\mathscr{G}(\boldsymbol{x}, G)$. It has dimension $n-m \ell$ and minimum distance $d \geq \ell+1$ [24, Chap. 12, p. 340]. In the special case where the roots $\boldsymbol{z}=\left(z_{0}, \ldots, z_{\ell-1}\right)$ of $G(X)$ are distinct and all belong to $\mathbb{F}_{q^{m}}$ then $\mathscr{G}(\boldsymbol{x}, G)$ admits a parity-check matrix $\boldsymbol{C}(\boldsymbol{z}, \boldsymbol{x})$ in Cauchy form [24, p. 345].

The scheme in [27] considers a Goppa code that admits a parity-check matrix that is both a Cauchy matrix and a block matrix where each block is dyadic. An $\ell \times \ell$ matrix $\boldsymbol{\Delta}=\left(\Delta_{i, j}\right)$ with $0 \leq i \leq \ell-1$ and $0 \leq j \leq \ell-1$ is dyadic if and only if $\Delta_{i, j}=h_{i \oplus j}$ where $\oplus$ is the bitwise exclusive-or on the binary representation of the indices and $\boldsymbol{h}=\left(h_{0}, \ldots, h_{\ell-1}\right)$ is the first row of $\boldsymbol{\Delta}$. Let $\boldsymbol{h}=\left(h_{0}, \ldots, h_{N-1}\right)$ be a vector of $\mathbb{F}_{q^{m}}^{N}$ with $\ell \leq N$. We denote by $\boldsymbol{\Delta}_{\ell}(\boldsymbol{h})=\left(\Delta_{i, j}\right)$ the $\ell \times N$ matrix such that $\Delta_{i, j}=h_{i \oplus j}$. One can easily observe that $\boldsymbol{\Delta}_{\ell}(\boldsymbol{h})$ is the juxtaposition of $N_{0}$ dyadic matrices of size $\ell \times \ell$ when $N=N_{0} \ell$ for some integer $N_{0}$. Proposition 7 proved in [27, Theorem 2] characterizes dyadic Cauchy matrices.

Proposition 7. A necessary and sufficient condition for $\boldsymbol{\Delta}_{\ell}(\boldsymbol{h})$ to be a Cauchy matrix $\boldsymbol{C}(\boldsymbol{z}, \boldsymbol{x})$ is that $\mathbb{F}_{q^{m}}$ is of characteristic 2 and for any $i, j$ in $\{0, \ldots, N-1\}$ we have:

$$
\frac{1}{h_{i \oplus j}}=\frac{1}{h_{j}}+\frac{1}{h_{i}}+\frac{1}{h_{0}} .
$$

Furthermore, for any $\theta \in \mathbb{F}_{q^{m}}$ and for any $z_{i}^{*}=1 / h_{i}+\theta$ and $x_{j}^{*}=1 / h_{j}+1 / h_{0}+\theta$, the Cauchy matrix $\boldsymbol{C}\left(\boldsymbol{z}^{*}, \boldsymbol{x}^{*}\right)$ is equal to $\boldsymbol{\Delta}_{\ell}(\boldsymbol{h})$.

Indeed, the public generator matrix $\boldsymbol{G}$ is a $k \times n$ block matrix where each block is an $\ell \times \ell$ dyadic matrix with $\ell$ being a power of 2 . The entries of $\boldsymbol{G}$ belong to $\mathbb{F}_{q}$ and the integers $k$ and $n$ are chosen such that $n=n_{0} \ell$ and $k=n-m \ell=\ell\left(n_{0}-m\right)$ where $n_{0}$ is some integer and $m$ defines the extension $\mathbb{F}_{q^{m}}$. The matrix $\boldsymbol{G}$ is obtained from a secret $\ell \times n$ block parity-check matrix $\boldsymbol{H}=\left(\boldsymbol{\Delta}_{\ell}\left(\boldsymbol{f}_{0}\right)|\cdots| \boldsymbol{\Delta}_{\ell}\left(\boldsymbol{f}_{n_{0}-1}\right)\right)$ where each block $\boldsymbol{\Delta}_{\ell}\left(\boldsymbol{f}_{j}\right)$ is an $\ell \times \ell$ dyadic matrix and for any $0 \leq j \leq n_{0}-1, \boldsymbol{f}_{j}$ is a vector of $\mathbb{F}_{q^{m}}^{\ell}$ such that $\boldsymbol{f}_{j}=\gamma_{j}\left(h_{\omega_{j} \ell \oplus d_{j}}, h_{\left(\omega_{j} \ell+1\right) \oplus d_{j}}, \ldots, h_{\left(\left(\omega_{j}+1\right) \ell-1\right) \oplus d_{j}}\right)$ where $\boldsymbol{h}=\left(h_{0}, \ldots, h_{N-1}\right)$ is a random vector of $\mathbb{F}_{q^{m}}^{N}$ that satisfies Equation (14) and such that $N=N_{0} \ell$ for 
some integer $N_{0} \gg n_{0}$. The integers $\omega_{j}, d_{j}$ are chosen such that $0 \leq \omega_{j} \leq N_{0}-1$ and $0 \leq d_{j} \leq \ell-1$. The coefficients $\gamma_{j}$ are non zero elements of $\mathbb{F}_{q^{m}}$. Note that the integers $\omega_{j}$ 's are different. The secret key consists then of the vectors $\boldsymbol{h}$, $\boldsymbol{\omega}=\left(\omega_{0}, \ldots, \omega_{n_{0}-1}\right), \boldsymbol{d}=\left(d_{0}, \ldots, d_{n_{0}-1}\right)$ and $\gamma=\left(\gamma_{0}, \ldots, \gamma_{n_{0}-1}\right)$.

\section{Proof of Proposition 4}

Lemma 3. Let $N=N_{0} \ell$ with $\ell=2^{e}$ for some integer $e$ and let $\boldsymbol{h}$ be a vector in $\mathbb{F}_{q^{m}}^{N}$ that satisfies Equation (14). Let $\mathscr{G}\left(\boldsymbol{a}^{*}, G\right)$ be the Goppa code such that $\boldsymbol{a}^{*}=\left(a_{0}^{*}, \ldots, a_{N-1}^{*}\right)$ is defined by $a_{j}^{*}=1 / h_{j}+1 / h_{0}$ for $0 \leq j \leq N-1$ and $G(X)=\prod_{i=0}^{\ell-1}\left(X-z_{i}\right)$ with $z_{i}=1 / h_{i}$. Then for any $i, j$ in $\{0, \ldots, N-1\}$ we have $a_{i \oplus j}^{*}=a_{i}^{*}+a_{j}^{*}$ and for any $0 \leq j \leq N_{0}-1$ and $0 \leq i, i^{\prime} \leq \ell-1$

$$
G\left(a_{j \ell+i}^{*}\right)^{-1}=\prod_{l=j \ell}^{(j+1) \ell-1} h_{l}
$$

Proof. The property that $a_{i \oplus j}^{*}=a_{i}^{*}+a_{j}^{*}$ comes from Equation (14). Furthermore, we have:

$$
G\left(a_{j \ell+i}^{*}\right)^{-1}=\prod_{\ell=0}^{\ell-1}\left(z_{\ell}-a_{j \ell+i}^{*}\right)^{-1}=\prod_{\ell=0}^{\ell-1}\left(1 / h_{\ell}+1 / h_{j \ell+i}+1 / h_{0}\right)^{-1}=\prod_{\ell=0}^{\ell-1} h_{j \ell+\ell}
$$

which terminates the proof.

We remark in particular that we have $G\left(a_{j \ell+i}^{*}\right)=G\left(a_{j \ell}^{*}\right)$ for any $0 \leq j \leq n_{0}-1$ and $0 \leq i \leq \ell-1$. The next lemma we give without proof shows that the action of a dyadic permutation can be simply characterized as a translation.

Lemma 4. Let $t$ and $d$ two integers such that $0 \leq d \leq \ell-1$. For any vector $\boldsymbol{v}=\left(v_{0}, \ldots, v_{\ell-1}\right)$, we have:

$$
\boldsymbol{v} \times \boldsymbol{\Delta}_{\ell}\left(\boldsymbol{b}_{d}\right)=\left(v_{d}, v_{1 \oplus d}, \ldots, v_{(\ell-1) \oplus d}\right)
$$

where the vector $\boldsymbol{b}_{d}=\left(b_{d, 0}, \ldots, b_{d, \ell-1}\right)$ is such that $b_{d, j}=0$ if $j \neq d$ and $b_{d, d}=1$.

We are now prepared to prove Proposition 4 Let $(\boldsymbol{h}, \boldsymbol{\omega}, \boldsymbol{d}, \boldsymbol{\gamma})$ be the private key and let $\boldsymbol{G}$ be the public generator matrix. We shall see that a parity-check matrix for the code generated by $\boldsymbol{G}$ is $\boldsymbol{V}_{\ell}(\boldsymbol{a}, \boldsymbol{\lambda})$ with $a_{j \ell+i}=a_{\left(\omega_{j} t+i\right) \oplus d_{\ell}}^{*}$ and $\lambda_{j \ell+i}=$ $\gamma_{j} G\left(a_{\omega_{j} t}^{*}\right)^{-1}$ where $\boldsymbol{a}^{*}$ and $G(X)$ are defined as in Lemma 3 . Indeed, we know that the code defined by the parity-check matrix $\boldsymbol{\Delta}_{\ell}(\boldsymbol{h})$ is also defined by the parity-check matrix $\boldsymbol{V}_{\ell}(\boldsymbol{a}, \boldsymbol{\lambda})$ where $\lambda_{j}=G\left(a_{j}\right)^{-1}$ for any $0 \leq j \leq N-1$. Recall from Lemma 3 that $G\left(a_{j \ell+i}\right)=G\left(a_{j \ell}\right)$ for any $0 \leq j \leq N_{0}-1$ and $0 \leq i \leq \ell-1$. The role of $\boldsymbol{\omega}$ is to pick $n_{0}$ dyadic blocks from $\boldsymbol{\Delta}_{\ell}(\boldsymbol{h})$. These blocks correspond to the columns $a_{\omega_{j} \ell}^{*}, \ldots, a_{\left(\omega_{j}+1\right) \ell-1}^{*}$ of $\boldsymbol{V}_{\ell}(\boldsymbol{a}, \boldsymbol{\lambda})$ when $j$ describes $\left\{1, \ldots, n_{0}\right\}$. These columns are then multiplied by a dyadic permutation matrix $\boldsymbol{\Delta}_{\ell}\left(\boldsymbol{b}_{d_{\ell}}\right)$ which leads to reorder the columns as $a_{\omega_{j} \ell \oplus d_{j}}, \ldots, a_{\left(\left(\omega_{j}+1\right) \ell-1\right) \oplus d_{j}}$ according to 
Lemma 4. Finally, each dyadic block is scaled by $\gamma_{j}$ which means that if we set $\lambda_{j \ell+i}=\gamma_{j} G\left(a_{\omega_{j} \ell}\right)^{-1}$ then $\boldsymbol{V}_{\ell}(\boldsymbol{a}, \boldsymbol{\lambda})$ is another parity-check matrix of the code generated by $\boldsymbol{G}$. We are now going to show that for any $0 \leq j \leq n_{0}-1$ and $0 \leq i, i^{\prime} \leq \ell-1$, we have the following equations:

$$
\begin{cases}\lambda_{j \ell+i} & =\lambda_{j \ell} \\ a_{j \ell+i}+a_{j \ell} & =a_{i}+a_{0} \\ a_{j \ell+i \oplus i^{\prime}} & =a_{j \ell+i}+a_{j \ell+i^{\prime}}+a_{j \ell}\end{cases}
$$

It is clear from Lemma 3 that $\lambda_{j \ell+i}=\lambda_{j \ell}$. On the other hand, $a_{j \ell+i}=a_{\left(\omega_{j} \ell+i\right) \oplus d_{j}}$ $=1 / h_{\left(\omega_{j} \ell+i\right) \oplus d_{j}}+1 / h_{0}$. From Equation (14) we thus have:

$$
\begin{aligned}
a_{j \ell+i} & =\frac{1}{h_{\omega_{j} \ell+i}}+\frac{1}{h_{d_{j}}}=\frac{1}{h_{\omega_{j} \ell}}+\frac{1}{h_{i}}+\frac{1}{h_{0}}+\frac{1}{h_{d_{j}}} \\
& =\frac{1}{h_{\omega_{j} \ell \oplus d_{j}}}+\frac{1}{h_{i}}+\frac{1}{h_{0}}=a_{j \ell}+\frac{1}{h_{i}}+\frac{1}{h_{0}} .
\end{aligned}
$$

We observe in particular that $a_{i}+a_{0}=1 / h_{i}+1 / h_{0}$ and since this quantity does not depend on $\ell$, this is equivalent to say that $a_{j \ell+i}+a_{j \ell}=a_{i}+a_{0}$. Before proving the third equation, we can first see that $a_{j \ell+i \oplus i^{\prime}}+a_{j \ell}=a_{i \oplus i^{\prime}}+a_{0}$. So if we know that $a_{i \oplus i^{\prime}}=a_{i}+a_{i^{\prime}}+a_{0}$ then we would get $a_{i \oplus i^{\prime}}=a_{j \ell+i}+a_{j \ell}+a_{i^{\prime}}$ which finally implies $a_{i \oplus i^{\prime}}=a_{j \ell+i}+a_{j \ell+i^{\prime}}+a_{0}$ that leads to the expected result. Now we have $a_{i \oplus i^{\prime}}=a_{\left(\omega_{1} \ell+i+i^{\prime}\right) \oplus d_{1}}=a_{\omega_{1} \ell+i+i^{\prime}}+a_{d_{1}}=a_{\omega_{1} \ell+i}+a_{i^{\prime}}+a_{d_{1}}=a_{\left(\omega_{1} \ell+i\right) \oplus d_{1}}+a_{i^{\prime}}$. Therefore we obtain:

$$
a_{i \oplus i^{\prime}}=a_{i}+a_{i^{\prime}}+a_{\omega_{1} \ell}+a_{d_{1}}+a_{\omega_{1} \ell}+a_{d_{1}}=a_{i}+a_{i^{\prime}}+a_{0} .
$$

